Toyokazu Hiramatsu

Nagoya Math. J.

Vol. 40 (1970), 173-192

\title{
EICHLER MAPS AND HYPERBOLIG FOURIER EXPANSION
}

\author{
TOYOKAZU HIRAMATSU
}

\section{§ 0. Introduction.}

In his lecture notes ([1, pp. 33-35], [2, pp. 145-152]), M. Eichler reduced 'quadratic' Hilbert modular forms of dimension $-k$ ( $k$ is a positive integer) to holomorphic automorphic forms of dimension $-2 k$ for the reproduced groups of indefinite ternary quadratic forms, by means of so-called Eichler maps.

On the other hand, H. Petersson ([5], [6]) introduced a Fourier expansion in a pair of hyperbolic fixed points for automorphic forms of real dimension with respect to fuchsian groups of the first kind, and constructed the Poincaré series at the pair of hyperbolic fixed points, and also calculated the inner product on the space of automorphic forms.

The purpose of the present paper is to prove the following theorem by combining the above two results:

Theorem. Let $O$ be a maximal order in an indefinite division quaternion algebra over $\boldsymbol{Q}$ and $\Gamma$ be the group of units in $O$. Then the n-th hyperbolic Fourier coefficient $c_{n}$ of the holomorphic automorphic form of dimension $-2 k$ for $\Gamma$ whih is obtained from a Hilbert modular form by the Eichler map $\tau$ is expressed as follows:

$$
\begin{aligned}
& c_{n}=(-1)^{n} \frac{1}{4 \log \varepsilon_{0}} q^{\pi i \frac{n}{4 \log \varepsilon_{0}}} e^{\frac{\pi^{2} n}{4 \log \varepsilon_{0}}} \Gamma\left(k+\frac{\pi n}{2 \log \varepsilon_{0}} i\right) \Gamma\left(k-\frac{\pi n}{2 \log \varepsilon_{0}} i\right) \times \\
& \times \sum_{\substack{\mu: \text { integersin } \\
\mu \equiv 0 \bmod \left(\frac{1}{\sqrt{p}}\right) \\
\mu>0 \text { or } \mu=0}}\left(\frac{\mu}{\mu}\right)^{\frac{\pi i}{\log \varepsilon_{0}} \frac{n}{4}} c_{\mu} \sum_{\nu=-\infty}^{\infty} i^{\nu} \frac{\varepsilon_{0}^{2 \nu}-\varepsilon_{0}^{-2 \nu}}{\nu-\pi i \frac{n}{2 \log \varepsilon_{0}}} J_{\nu}\left(4 \pi i\left(\frac{N(\mu)}{q}\right)^{\frac{1}{2}}\right) \times \\
& \times \frac{1}{\Gamma\left(k+\frac{\nu}{2}+\pi i \frac{n}{4 \log \varepsilon_{0}}\right) \Gamma\left(k-\frac{\nu}{2}-\pi i \frac{n}{4 \log \varepsilon_{0}}\right)},
\end{aligned}
$$

Received May 22, 1969. 
where $c_{\mu}$ denote parabolic Fourier coefficients of the Hilbert modular form stated above.

During the preparation of the present paper the author received many useful bits of advice from Professors T. Kubota and H. Shimizu. The author wishes to express his hearty thanks to them.

Notation. $\boldsymbol{Z}, \boldsymbol{Q}, \boldsymbol{R}$ and $\boldsymbol{C}$ denote respectively the ring of rational integers, the rational number field, the real number field and the complex number field. If $R$ is a ring, $M_{2}(R)$ denotes the ring of all matrices of degree 2 with coefficients in $R$.

\section{$\S 1$. Eichler maps $\tau$.}

Let $A$ be an indefinite division quaternion algebra over $\boldsymbol{Q}$, i.e., a division algebra over $\boldsymbol{Q}$ such that $A \otimes \boldsymbol{R}$ is isomorphic to $M_{2}(\boldsymbol{R})$ and let $\{1, \omega$, $\Omega, \omega \Omega$ \} be a basis of $A$ over $\boldsymbol{Q}$ such that $\omega^{2}=p, \Omega^{2}=-q$ and $\omega \Omega=-\Omega \omega$, where $p$ and $q$ are distinct prime numbers with the Legendre symbol $\left(\frac{-q}{p}\right)=-1$. We put

$$
F=\boldsymbol{Q}(\sqrt{p}) .
$$

Then, identifying $F$ with the real quadratic subfield $\boldsymbol{Q}(\omega)$ in $A$, We may write $A=F+F \Omega$ and we have $\alpha \Omega=\Omega \bar{\alpha}$ for all $\alpha \in F, \bar{\alpha}$ being the conjugate of $\alpha$ with respect to $F$. This expression gives the faithful representation $\chi$ of $A$ into $M_{2}(F)$ :

$$
\chi(\xi)=\left(\begin{array}{cc}
\xi_{1} & \xi_{2} \\
-q \bar{\xi}_{2} & \bar{\xi}_{1}
\end{array}\right)
$$

for $\xi=\xi_{1}+\xi_{2} \Omega \in A$ with $\xi_{i} \in F$. we see easily that the conjugate of $\xi$ is $\xi^{\prime}=\bar{\xi}_{1}-\bar{\xi}_{2} \Omega$, the trace of $\xi$ is $\operatorname{tr}(\xi)=\xi+\xi^{\prime}=\xi_{1}+\bar{\xi}_{1}=\operatorname{tr} \chi(\xi)$ and the norm of $\xi$ is $n(\xi)=\xi \xi^{\prime}=\xi_{1} \bar{\xi}_{1}+q \xi_{2} \bar{\xi}_{2}=\operatorname{det} \chi(\xi)$.

Let $G=S L(2, \boldsymbol{R})$ and $K$ be a maximal compact subgroup of $G$ (i.e. $K=S O(2))$. Let $O$ be the ring consisting of all elements in the form $\xi_{1}+\xi_{2} \Omega$, where $\xi_{1}, \xi_{2}$ are in the set of all integral algebraic numbers in $F$ and let $\Gamma^{p \times q 1)}$ be the group of (proper) units in $O$. From now on we assume that $p \equiv 1 \bmod 4$. Then $O$ is the maximal order in $A$. We know that $\Gamma$, regarded as a subgroup of $G$, is a fuchsian group of the first kind and has a compact fundamental domain $\Gamma \backslash G / K$.

Let $V=M_{2}(\boldsymbol{R}) \times M_{2}(\boldsymbol{R})$, and define $\rho: G \rightarrow G L(V)$ by

1) When there is no danger of confusion we may write $\Gamma$ instead of $\Gamma^{p \times q}$. 


$$
\rho(g)\left(\zeta_{1}, \zeta_{2}\right)=\left(\sigma_{1}(g) \zeta_{1}, \sigma_{2}(g) \zeta_{2}\right)
$$

for each $g$ in $G$, where $\left(\zeta_{1}, \zeta_{2}\right) \in V$ and each $\sigma_{i}(i=1,2)$ denotes an irreducible continuous representation of $G$ (i.e. $\rho=\sigma_{1} \oplus \sigma_{2}$ ). Let $\rho_{n}$ be the symmetric tensor representation of degree $n$ of $G L(2, \boldsymbol{R})$. Then, it is well known that the restriction $\rho_{n} \mid G$, which we denote again by $\rho_{n}$, exhaust all irreducible continuous representations of $G$ :

$$
\rho_{n}: G \rightarrow S L(n+1, \boldsymbol{R}) \quad(n=0,1,2, \cdots) .
$$

Therefore each of $\sigma_{i}(i=1,2)$ takes the following form:

$$
\left\{\begin{array}{l}
\sigma_{1}=\beta^{-1} i d . \beta, \\
\sigma_{2}=\alpha^{-1} i d . \alpha,
\end{array}\right.
$$

where $\alpha, \beta \in G L(2, \boldsymbol{R})$ and $i d$. denotes the identical representation. Hereafter we assume that $\beta=\left(\begin{array}{ll}1 & 0 \\ 0 & 1\end{array}\right)$.

Now we put

$$
G_{0}=G \times G, K_{0}=K \times \alpha^{-1} K \alpha .
$$

Then $K_{0}$ is a maximal compact subgroup of $G_{0}$ and $\rho(G) \subset G_{0}, \rho(K) \subset K_{0}$. Hence the homomorphism $\rho$ induces a map $\tau$ of the quotient space $G / K$ into the quotient space $G_{0} / K_{0}$ :

$$
\tau: G / K=\mathfrak{S}^{+} \rightarrow \mathfrak{S g}^{+} \times \mathfrak{S}^{+}=G / K \times G / \alpha^{-1} K \alpha=G_{0} / K_{0},
$$

Where $\mathfrak{S}^{+}$denotes the complex upper half-plane. Let $\Gamma(\sqrt{p})$ be the Hilbert modular group of the real quadratic field $F$. Then, if $\rho$ satisfies the condition

$$
\rho(\Gamma) \subset \Gamma(\sqrt{p})
$$

as operator, the map $\tau$ induced from $\rho$ will be called Eichler map of $\rho$.

Lemma 1. The homomorphism $\rho$ satisfies the condition $\rho(\Gamma) \subset \Gamma(\sqrt{p})$ as operator if and only if $\alpha=\left(\begin{array}{rr}0 & 1 \\ -q & 0\end{array}\right) \in O$.

Proof. If $\rho$ satisfies the condition $\rho(\Gamma) \subset \Gamma(\sqrt{p})$ as operator, then $\alpha \gamma^{\prime}=\gamma \alpha$ for all $\gamma \in \Gamma$. Hence, putting $\alpha=\left(\begin{array}{ll}a & b \\ c & d\end{array}\right), \gamma=\left(\begin{array}{cc}\xi_{1} & \xi_{2} \\ -q \bar{\xi}_{2} & \bar{\xi}_{1}\end{array}\right)$, we have the following relations: 


$$
\left\{\begin{array}{l}
a \bar{\xi}_{1}-b q \xi_{2}=a \xi_{1}+c \xi_{2}, \\
a \bar{\xi}_{2}=d \xi_{2} \\
c \bar{\xi}_{2}-d \xi_{1}=-b q \bar{\xi}_{2}+d \bar{\xi}_{1} .
\end{array}\right.
$$

If we take $\gamma=\left(\begin{array}{ll}\varepsilon_{0}^{2} & 0 \\ 0 & \varepsilon_{0}^{-2}\end{array}\right)$, where $\varepsilon_{0}$ denotes a fundamental unit of the real quadratic field $F$, then the third relation implies that $d \varepsilon_{0}^{4}=d$. If $d \neq 0$ then $\varepsilon_{0}^{4}=1$. This is impossible. Hence we have $d=0$. Similarly, $a=0$. Therefore we have $-b q \xi_{2}=c \xi_{2}$. There exists an element of $\Gamma$ such that $\xi_{2} \neq 0$. In fact, otherwise, there would be only two primitive hyperbolic conjugacy classes in $\Gamma$, namely, $\left\{\left(\begin{array}{cc}\varepsilon_{0}^{2} & 0 \\ 0 & \varepsilon_{0}^{-2}\end{array}\right)\right\}$ and $\left\{\left(\begin{array}{cc}\varepsilon_{0}^{-2} & 0 \\ 0 & \varepsilon_{0}^{2}\end{array}\right)\right\}$. But this contradicts the fact that the group $\Gamma$ has an infinite number of primitive hyperbolic conjugacy classes. Hence $c=-b q$; and this means $\alpha=\left(\begin{array}{cc}0 & b \\ -b q & 0\end{array}\right)$. Therefore, as the linear fractional transformation, $\alpha$ takes the form $\left(\begin{array}{rr}0 & 1 \\ -q & 0\end{array}\right)$. And vice versa.

From Lemma 1, the homomorphism $\rho$ which induced Eichler map $\tau$ can be written in the form

$$
\left\{\begin{array}{l}
\rho=i d . \oplus \overline{i d} ., \overline{i d} .=\alpha^{-1} i d . \alpha \\
\alpha=\left(\begin{array}{rr}
0 & 1 \\
-q & 0
\end{array}\right)
\end{array}\right.
$$

Since $K$ is the isotropy subgroup of the point $i\left(\in \mathfrak{H}^{+}\right)$in $G, \alpha^{-1} K \alpha$ is that of the point $\alpha(i)$ in $G$; and the map $\tau$ takes the following explicite form:

$$
\tau: \mathfrak{S}^{+} \ni z \longrightarrow(z, \alpha(z))=\left(z, \frac{-1}{q z}\right) \in \mathfrak{S}^{+} \times \mathfrak{S}^{+} .
$$

Obviously the map $\tau$ is holomorphic and by the map $\tau$, a compact fundamental domain $\Gamma \backslash G / K$ for the group $\Gamma$ is imbedded holomorphic into a noncompact but volume-finite fundamental domain $\Gamma(\sqrt{p}) \backslash G_{0} / K_{0}$ for the group $\Gamma(\sqrt{p})$.

\section{§ 2. Automorphic forms associated with Eichler maps $\tau$}

Given a group $\Gamma^{p \times q}$ as descrived in $\S 1$, then there exists the Hilbert modular group $\Gamma(\sqrt{p})$. It satisfies the condition $\rho(\Gamma) \subset \Gamma(\sqrt{p})$, namely,

$$
\rho(\gamma)=\left(\left(\begin{array}{cc}
\xi_{1} & \xi_{2} \\
-q \bar{\xi}_{2} & \bar{\xi}_{1}
\end{array}\right),\left(\begin{array}{rr}
0 & 1 \\
-q & 0
\end{array}\right)^{-1}\left(\begin{array}{rr}
\xi_{1} & \xi_{2} \\
-q \bar{\xi}_{2} & \bar{\xi}_{1}
\end{array}\right)\left(\begin{array}{rr}
0 & 1 \\
-q & 0
\end{array}\right)\right)
$$




$$
\begin{aligned}
& =\left(\left(\begin{array}{cc}
\xi_{1} & \xi_{2} \\
-q \bar{\xi}_{2} & \bar{\xi}_{1}
\end{array}\right), \overline{\left.\left(\begin{array}{cc}
\xi_{1} & \bar{\xi}_{2} \\
-q \bar{\xi}_{2} & \bar{\xi}_{1}
\end{array}\right)\right)}\right. \\
& \in \Gamma(\sqrt{p})
\end{aligned}
$$

for each $\gamma=\left(\begin{array}{rr}\xi_{1} & \xi_{2} \\ -q \bar{\xi}_{2} & \bar{\xi}_{1}\end{array}\right) \in \Gamma$.

Now let $k$ be a positive integer and we denote by $S_{k}(\Gamma(\sqrt{p}))$, the space of all (holomorphic) Hilbert modular forms of dimension $-k$ for the group $\Gamma(\sqrt{p})$. With each element $F(\boldsymbol{z})=F\left(z_{1}, z_{2}\right)$ in the space $S_{k}(\Gamma(\sqrt{p}))$ we associate a function $f(z)$ in the following manner:

$$
\begin{aligned}
& \left(\tau^{*} \circ F\right)(z)=F(\tau(z)), \quad z \in \mathfrak{S}^{+}, \\
& f(z)=z^{-k}\left(\tau^{*} \circ F\right)(z) .
\end{aligned}
$$

Then, under the condition $f(z) \neq$ constant, $f(z)$ is a (non-trivial) holomorphic function in $\mathfrak{S}^{+}$and satisfies the transformation law

$$
f(\gamma(z))=\left(-q \bar{\xi}_{2} z+\bar{\xi}_{1}\right)^{2 k} f(z)
$$

for all $\gamma=\left(\begin{array}{cc}\xi_{1} & \xi_{2} \\ -q \bar{\xi}_{2} & \bar{\xi}_{1}\end{array}\right) \in \Gamma$. The holomorphic property of $f(z)$ is immediate from that of the map $\tau$. The later part is shown as follows:

$$
\begin{aligned}
f(\gamma(z)) & =\gamma(z)^{-k}\left(\tau^{*} \circ F\right)(\gamma(z)) \\
& =\gamma(z)^{-k} F(\rho(\gamma)(\tau(z))),
\end{aligned}
$$

and for each $\sigma=\left(\begin{array}{ll}a & b \\ c & d\end{array}\right) \in \Gamma(\sqrt{p})$, putting

$$
j(\sigma, \boldsymbol{z})=\left\{\left(c z_{1}+d\right)\left(\bar{c} z_{2}+\bar{d}\right)\right\}^{-k},
$$

we have

$$
\begin{aligned}
F(\rho(\gamma)(\tau(z))) & =j(\rho(\gamma), \tau(z))^{-1} F(\tau(z)) \\
& =\left\{\left(q \bar{\xi}_{2} z+\bar{\xi}_{1}\right)\left(-q \xi_{2}\left(\frac{-1}{q z}\right)+\xi_{1}\right)\right\}^{k}\left(\tau^{*} \circ F\right)(z) \\
& =\left(-q \bar{\xi}_{2} z+\bar{\xi}_{1}\right)^{2 k} \gamma(z)^{k} z^{-k}\left(\tau^{*} \circ F\right)(z) \\
& =\left(-q \bar{\xi}_{2} z+\bar{\xi}_{1}\right)^{2 k} \gamma(z)^{k} f(z) .
\end{aligned}
$$

This implies our assertion. Therefore, under the condition $f(z) \neq$ constant, $f(z)$ is a holomorphic automorphic form of dimension $-2 k$ for the group $\Gamma$. 
The Hilbert modular form has parabolic Fourier expansion of the form

$$
\begin{aligned}
F\left(z_{1}, z_{2}\right)= & \sum_{\substack{\mu: \text { integers in } F \\
\mu \equiv 0 \bmod \left(\frac{1}{\sqrt{p}}\right) \\
\mu>0 \text { or } \mu=0}} c_{\mu} e^{2 \pi i t r(\mu z)}, \\
&
\end{aligned}
$$

where $\operatorname{tr}(\mu \boldsymbol{z})=\mu z_{1}+\bar{\mu} z_{2}$ and $\mu>0$ implies that $\mu$ is totally positive. We put $K_{\mu}=\left\{\varepsilon_{0}^{2 n} \mu \mid n \in Z\right\}$ and denotes by $\{(\mu)\}$ a set of representatives of $\left\{K_{\mu}\right\}$. Then, the form $f(z)$ has the following expansion:

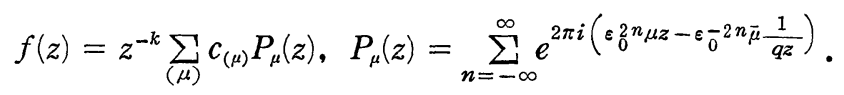

This expansion for $f(z)$ was introduced by Eichler ([1, p. 35], [2, p. 151]).

Remark. The series $P_{\mu}(z)$ converges absolutely, uniformly on $\mathfrak{S}^{+} \times \mathfrak{S}^{+}$. In fact, let $z_{j}=x_{j}+i y_{j}$ and $y_{j} \geqq d_{j}>0 \quad(j=1,2), d_{j}$ being a constant. Then,

$$
\begin{aligned}
\mid e^{2 \pi i\left(\varepsilon_{0}^{2 n} \mu z_{1}+\varepsilon_{0}^{\left.-2 n \bar{\mu} z_{2}\right)} \mid\right.}= & e^{-2 \pi \varepsilon_{0}^{2 n} \mu y_{1}} e^{-2 \pi \varepsilon_{0}^{-2 n \bar{\mu} y_{2}}} \\
& <\left\{\begin{array}{l}
e^{2 \pi \varepsilon_{0}^{-2 n \bar{\mu} y_{2}} \leqq}\left(\frac{1}{\varepsilon_{0}}\right)^{4 \pi \bar{\mu} d_{2} n}, \text { if } n \geqq 0, \\
e^{2 \pi \varepsilon_{0}^{2 n} n y_{1}} \leqq\left(\frac{1}{\varepsilon_{0}}\right)^{-4 \pi \mu d_{1} n}, \text { if } n<0,
\end{array}\right.
\end{aligned}
$$

where $\varepsilon_{0}$ satisfies $\varepsilon_{0}>1$. This implies the remark.

Now, since the series $P_{\mu}(z)$ is invariant under the transformation $\left(\begin{array}{ll}\varepsilon_{0}^{2} & 0 \\ 0 & \varepsilon_{0}^{-2}\end{array}\right)$ in the group $\Gamma$, the above expansion for $f(\boldsymbol{z})$ may be regarded as an expansion at the pair of hyperbolic fixed points $\{0, \infty\}$. But unfortunately, this expansion is not unique in general. In fact, let $\mathfrak{S}_{2 k}(\Gamma)$ be the space of all holomorphic automorphic forms of dimension $-2 k$ for the group $\Gamma$. For each function $F(z)$ in the space $S_{k}(\Gamma(\sqrt{p}))$, let $f(z)$ be the function associated with it by the Eichler map $\tau$. Then, $T: F(\boldsymbol{z}) \longrightarrow f(z)$ gives a $\boldsymbol{C}$-linear map of the space $S_{k}(\Gamma(\sqrt{p}))$ to the space $\mathfrak{S}_{2 k}(\Gamma)$. If $P_{\mu}$-expansion is unique, then we have

$$
f(z) \equiv 0 \Longrightarrow \text { all } c_{(\mu)}=0 \Longrightarrow F(z) \equiv 0 .
$$

Hence the map $T$ is one-to-one, so that

$$
\operatorname{dim}_{\boldsymbol{C}} S_{k}(\Gamma(\sqrt{p}))=\operatorname{dim}_{\boldsymbol{C}} T\left(S_{k}(\Gamma(\sqrt{p})) \leqq \operatorname{dim}_{\boldsymbol{C}} \Im_{2 k}(\Gamma) .\right.
$$


On the other hand, we know that

$$
\operatorname{dim}_{\boldsymbol{C}} S_{k}(\Gamma(\sqrt{p}))=a k^{2}+b k+c \quad(a>0)
$$

by Shimizu [8, p. 63] and

$$
\operatorname{dim}_{C} \Im_{2 k}(\Gamma)=d k+e .
$$

Therefore (1) is impossible for sufficiently large $k$.

We give some examples and remark.

Example 1. Let $k$ be an even integer and let $G_{-k}(\boldsymbol{z})$ be an Eisenstein series of dimension $-k$ for the group $\Gamma(\sqrt{p})$. Then the function $G_{-k}(\boldsymbol{z})$ has the following Fourier expansion:

$$
G_{-k}(\boldsymbol{z})=1+\sum_{\substack{\mu: \text { integers in } F \\ \mu \equiv 1 \bmod \left(\frac{1}{\sqrt{p}}\right) \\ \mu>0}} c_{\mu} e^{2 \pi i t r(\mu z)},
$$

where

$$
\begin{aligned}
& c_{\mu}=\frac{(2 \pi)^{2 k} \sqrt{p}}{\{(k-1) !\}^{2} p^{k} \zeta_{F}(k)} \sum_{\substack{(\nu): \text { integral ideals in } F \\
(\nu) \mid(\sqrt{p} \mu)}}|N(\nu)|^{k-1}, \\
& \zeta_{F}(s)=\sum_{(\nu): \text { integral ideals in }}|N(\nu)|^{-s} .
\end{aligned}
$$

Therefore we have

$$
T\left(G_{-k}(\boldsymbol{z})\right)=f_{-2 k}(z)=z^{-k}\left\{1+\sum_{\mu} c_{\mu} e^{2 \pi i\left(\mu z-\bar{\mu} \frac{1}{q z}\right)}\right\}
$$

Put $z=i\left(\in \mathfrak{S}^{+}\right)$. Then $2 \pi i\left(\mu z-\bar{\mu} \frac{1}{q z}\right)$ is equal to a real number; and also $c_{\mu}>0$. Hence $f_{-2 k}(i) \neq 0$, i.e., $f_{-2 k}(z) \not$ constant.

Example 2. As a numerical example, consider the quaternion algebra $A$ over $\boldsymbol{Q}$ with a basis $\{1, \omega, \Omega, \omega \Omega\}$ such that $\omega^{2}=5, \Omega^{2}=-2$. Then the only characteristic primes for this $A / Q$ are 2 and 5 ; and $\mathfrak{S}^{+} / \Gamma^{5 \times 2}$ has four elliptic fixed points of order 3 and its genus is zero. Hence, $\operatorname{dim} \widetilde{\Im}_{8}\left(\Gamma^{5 \times 2}\right)=1$. On the other hand, $\operatorname{dim} S_{4}(\Gamma(\sqrt{5}))=2$ by Gundlach [3, p. 382], and

$$
\begin{aligned}
T\left(G_{-4}(\boldsymbol{z})\right)=f_{-8}(z) & =z^{-4}\left\{1+2^{4} \cdot 3 \cdot 5 \sum_{\mu} \sum_{(\nu) \mid \sqrt{5} \mu}|N(\nu)|^{3} e^{2 \pi i\left(\mu z-\bar{\mu} \frac{1}{2 z}\right)}\right\} \\
& \neq \text { constant. }
\end{aligned}
$$


Therefore a function obtained from the cusp form in $S_{4}(\Gamma(\sqrt{5}))$ by the map $T$ is linearly dependent to the function $f_{8}(z)$ or identical with zero. But its decision would be difficult.

Remark. It would be of great interest to determine the kernel of homomorpnism $T: \sum_{k=0}^{\infty} S_{k}(\Gamma(\sqrt{p})) \longrightarrow \sum_{k=0}^{\infty} \mathbb{S}_{2 k}\left(\Gamma^{p \times q}\right)$.

Example 3. It is not true in general that any form for the group $\Gamma^{p \times q}$ is always obtained from some form for the group $\Gamma(\sqrt{p})$ by the map $T$. For example,

$$
\operatorname{dim}_{\boldsymbol{C}} \Im_{8}\left(\Gamma^{5 \times 7}\right)=14 \text { and } \operatorname{dim}_{\boldsymbol{C}} S_{4}(\Gamma(\sqrt{5}))=2 .
$$

\section{§3. Hyperbolic Poincaré series}

This section is essentially based upon the work of Petersson [5] and we shall describe those parts of it applicable to our case.

Let $A_{0}$ be an indefinite division quaternion algebra over $\boldsymbol{Q}$ and let $\{1, \omega, \Omega, \omega \Omega\}$ be a basis of $A_{0}$ over $\boldsymbol{Q}$ such that $\omega^{2}=p_{0}, \Omega^{2}=-q_{0}$ and $\omega \Omega=-\Omega \omega$, where $p_{0}$ is a positive integer, $q_{0}$ is a integer with Jacobi symbol $\left(\frac{-q_{0}}{p_{0}}\right)=-1$. we put

$$
O_{1}=\left\{\left(\begin{array}{cc}
\xi_{1} & \xi_{2} \\
-q_{0} \bar{\xi}_{2} & \bar{\xi}_{1}
\end{array}\right) \mid \xi_{1}, \xi_{2}: \text { integral algebraic numbers in } F_{0}=\boldsymbol{Q}\left(\sqrt{p_{0}}\right)\right\}
$$

Then $O_{1}$ is the order in $A_{0}$. Let $O_{0}$ be a maximal order in $A_{0}$ such that $O_{0} \supseteq O_{1}$ and let $\Gamma_{0}{ }^{p_{0} \times q_{0}}$ be the group of (proper) units in $O_{0}{ }^{2}$ ) Then the fuchsian group $\Gamma_{0}$ has a compact fundamental domain $\Gamma_{0} \mid G / K$ and contains the hyperbolic element $\left(\begin{array}{ll}\varepsilon_{0}^{2} & 0 \\ 0 & \varepsilon_{0}^{-2}\end{array}\right)$ which has fixed points $\{0, \infty\}$, where $\varepsilon_{0}$ denotes a fundamental unit of $F_{0}$ such that $\varepsilon_{0}>1$.

Remark. Let $O^{*}$ be any maximal order in $A_{0}$. Then, since $A_{0}$ is indefinite, $O^{*}$ is conjugate with $O_{0}$, i.e., there exists an element $\alpha$ in $A_{0}$ such that $n(\alpha) \neq 0$ and $O^{*}=\alpha^{-1} O_{0} \alpha$. Therefore the unit group $\Gamma^{*}$ in $O^{*}$ has the same genus and signature as $\Gamma_{0}$ and contains the hyperbolic element $\alpha^{-1}\left(\begin{array}{ll}\varepsilon_{0}^{2} & 0 \\ 0 & \varepsilon_{0}^{-2}\end{array}\right) \alpha$ which fixes points $\{0, \infty\}$. The case of $\Gamma^{*}$ can be treated by a similar way as in the following method for $\Gamma_{0}$, so that this case is omitted here.

2) The unit group in $O_{1}$ is commensurable with $\Gamma_{0}$. 
First of all, we shall induce a Fourier expansion for the function $f(z)$ in $\mathfrak{S}_{2 k}(\Gamma)$ ( $k$ is a positive integer) at the pair of hyperbolic fixed points $\{0, \infty\}$ which are fixed points of $\left(\begin{array}{cc}\varepsilon_{0}^{2} & 0 \\ 0 & \varepsilon_{0}^{-2}\end{array}\right)$. We put $g(z)=z^{k} f(z)$. Then the function $g(z)$ is invariant under the transformation $\sigma=\left(\begin{array}{cc}\varepsilon_{0}^{2} & 0 \\ 0 & \varepsilon_{0}^{-2}\end{array}\right)$ in the group $\Gamma_{0}$. Put

$$
t=\frac{\log z}{\log \varepsilon_{0}^{4}}, 0<\arg z<\pi .
$$

Then, since $\sigma(t)=t+1$, a function $\tilde{g}(t)$ considered $g(z)$ to be a function of $t$ is invariant under the translation $t \rightarrow t+1$. Hence, if we put $q=e^{2 \pi i t}$, there exists a function $\hat{g}(q)$ holomorphic in the domain

$$
e^{-\frac{2 \pi^{2}}{\log 80^{4}}<|q|<1}
$$

such that $\tilde{g}(t)=\hat{g}(q)$. Therefore the function $\hat{g}(q)$ has a Laurent expansion in $q$ :

$$
\hat{g}(q)=\sum_{n=-\infty}^{\infty} c_{n} q^{n} .
$$

As mentioned above, the form $f(z)$ in $\widetilde{S}_{2 k}\left(\Gamma_{0}\right)$ has the following expansion:

$$
\begin{aligned}
f(z) & =z^{-k} \sum_{n=-\infty}^{\infty} c_{n} e^{2 \pi i \frac{\log z}{\log \varepsilon_{0} 4^{4}}} \\
& =z^{-k} \sum_{n=-\infty}^{\infty} c_{n} z^{\pi i \frac{n}{2 \log \varepsilon_{0}}} .
\end{aligned}
$$

This expansion is called hyperbolic Fourier expansion for $f(z)$, briefly HFE for $f(z)$.

Next we shall introduce a Poincaré series with respect to $\sigma$.

(i) Definition and transformation law.

Let $Z_{0, \infty}$ be the subgroup of $\Gamma_{0}$ generated by $\sigma=\left(\begin{array}{ll}\varepsilon_{0}^{2} & 0 \\ 0 & \varepsilon_{0}^{-2}\end{array}\right)$ and let $\Re\left(\Gamma_{0}\right)$ be a system of right coset representatives of $\Gamma_{0} \bmod Z_{0, \infty}$. Then the Poincaré series for $\sigma$ is defined as

$$
\Xi_{-2 k}\left(z ; \Gamma_{0}, \nu\right)=Z_{-2 k}(z ; \nu)=\sum_{\substack{L \in \Re\left(\Gamma_{1}\right) \\
L=\left(\begin{array}{c}
\alpha_{1} \alpha_{2} \\
\beta_{1} \beta_{2}
\end{array}\right)}} \frac{e^{2 \pi i \nu \frac{\log L(z)}{\log 0_{0}}}}{\left(\alpha_{1} z+\alpha_{2}\right)^{k}\left(\beta_{1} z+\beta_{2}\right)^{k}},
$$

where $k>1,0<\arg L(z)<\pi$ and $\nu$ is a parameter extended over all inte- 
gers. Obviously the summation is independent of the coset representatives chosen.

For each $r \in \Gamma_{0}, \Re\left(\Gamma_{0}\right) \gamma$ is also a system of coset representatives; and putting

$$
\begin{aligned}
& \varphi(z ; L)=\left(\alpha_{1} z+\alpha_{2}\right)^{k}\left(\beta_{1} z+\beta_{2}\right)^{k}, \\
& \gamma=\left(\begin{array}{ll}
a & b \\
c & d
\end{array}\right) \text { and } L r=\left(\begin{array}{ll}
\alpha_{1}^{\prime} & \alpha_{2}^{\prime} \\
\beta_{1}^{\prime} & \beta_{2}^{\prime}
\end{array}\right),
\end{aligned}
$$

we have

$$
\begin{aligned}
& \varphi(\gamma(z) ; L)=\left(\alpha_{1} \frac{a z+b}{c z+d}+\alpha_{2}\right)^{k}\left(\beta_{1} \frac{a z+b}{c z+d}+\beta_{2}\right)^{k} \\
& \quad=(c z+d)^{-2 k}\left\{\left(\alpha_{1} a+\alpha_{2} c\right) z+\left(\alpha_{1} b+\alpha_{2} d\right)\right\}^{k}\left\{\left(\beta_{1} a+\beta_{2} c\right) z+\left(\beta_{1} b+\beta_{2} d\right)\right\}^{k} \\
& \quad=(c z+d)^{-2 k}\left(\alpha_{1}^{\prime} z+\alpha_{2}^{\prime}\right)^{k}\left(\beta_{1}^{\prime} z+\beta_{2}^{\prime}\right)^{k} .
\end{aligned}
$$

Therefore we obtain the following transformation law:

$$
\Xi_{-2 k}(\gamma(z) ; \nu)=(c z+d)^{2 k} \Xi_{-2 k}(z ; \nu)
$$

for all $r=\left(\begin{array}{ll}a & b \\ c & d\end{array}\right) \in \Gamma_{0}$.

(ii) Convergence of the series $\Xi_{-2 k}\left(z ; \Gamma_{0}, \nu\right)$.

$1^{\circ}$. Convergence of the series $\sum_{L}\left|\beta_{1} z+\beta_{2}\right|^{-2 k}\left(L=\left(\begin{array}{cc}* & * \\ \beta_{1} & \beta_{2}\end{array}\right) \in \Re\left(\Gamma_{0}\right), k>1\right)$.

In the following, we shall give a brief outline of the proof. ${ }^{3}$ ) We fix a point $z_{0}\left(\in \mathfrak{S}^{+}\right)$and take a constant $\rho_{0}$ such that

$$
0<\rho_{0}<\min _{\substack{r \in \Gamma_{0} \\ \gamma\left(z_{0}\right) \neq z_{0}}}\left|\gamma\left(z_{0}\right)-z_{0}\right|_{H}
$$

where ||$_{H}$ denotes the hyperbolic distance; and we put

$$
\Re_{0}=\left\{z \in \mathfrak{S}^{+}|| z-\left.z_{0}\right|_{H} \leqq \rho_{0}\right\} .
$$

For a suitable choice of coset representatives $\mathfrak{R}\left(\Gamma_{0}\right)$, each element in $\mathfrak{R}\left(\Gamma_{0}\right)$ may satisfy the condition

$$
-\log \varepsilon_{0}^{2}<\log \left|L\left(z_{0}\right)\right| \leqq \log \varepsilon_{0}^{2} .
$$

Then, there exist two constants $c_{1}$ and $c_{2}$ satisfying

$$
c_{1} e^{-|L(z)-i|_{H}}<\left|\beta_{1} z+\beta_{2}\right|^{-2}<c_{2} e^{-|L(z)-i|_{H}}
$$

3) As for full detail of the proof, cf. Petersson [5, pp. 144-146]. 
for all $L=\left(\begin{array}{cc}* & * \\ \beta_{1} & \beta_{2}\end{array}\right) \in \Re\left(\Gamma_{0}\right)$, and all $z \in \mathfrak{\Re}_{0}$.

Next we take a constant $R$ such that $R>\varepsilon_{0}^{2} e^{\rho_{0}}$; and put

$$
\begin{aligned}
& \Re_{R}=\left\{z \in \mathfrak{S}^{+}|| z-\left.i\right|_{H} \leqq R\right\}, \\
& \mathfrak{D}_{R}=\Re_{R} \cap\left\{z \in \mathfrak{S}^{+}|| \log |z| \mid \leqq \log \varepsilon_{0}^{2} e^{\rho_{0}}\right\}
\end{aligned}
$$

and

$$
\mathfrak{N}_{R}=\left\{L \in \Re\left(\Gamma_{0}\right) \mid L z_{0} \in \mathfrak{D}_{R}\right\} .
$$

Then, the number $m_{R}$ of elements contained in the set $\mathfrak{N}_{R}$ is finite and moreover there exists a constant $c_{3}$ satisfying the following condition:

$$
m_{R}<c_{3} e^{R} .
$$

Finally we take a constant $R_{0}$ such that $R_{0}>\varepsilon_{0}^{2} e^{\rho_{0}}$ and $R_{0}>\rho_{0}$; and put

$$
\mathfrak{\Im}_{n}=\mathfrak{N}_{n R_{0}}-\mathfrak{A}_{(n-1) R_{0}}(n \geqq 2), \mathfrak{\Im}_{1}=\mathfrak{N}_{R_{0}} .
$$

Then, by (2) and (3), there exists a constant $c_{4}$ which is independent of $n(\geqq 2)$ and satisfies the condition

$$
\sum_{L \in \Im_{n}}\left|\beta_{1} z+\beta_{2}\right|^{-2 k}<c_{4} e^{(1-k) R_{0} n} \quad(n \geqq 2) .
$$

This implies

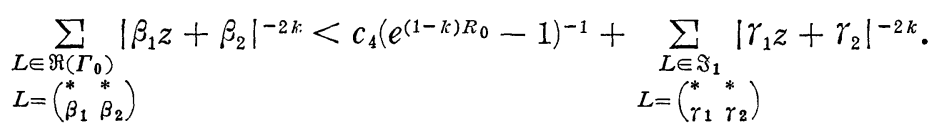

Therefore the series $\sum_{L \in \Re\left(T_{0}\right)}\left|\beta_{1} z+\beta_{2}\right|^{-2 k}$ converges over $\Re_{0}$, since the sum $\sum_{L \in \Im_{1}}$ is finite.

$2^{\circ}$. Convergence of the series $\sum_{L}\left|\alpha_{1} z+\alpha_{2}\right|^{-k}\left|\beta_{1} z+\beta_{2}\right|^{-k}\left(L=\left(\begin{array}{cc}\alpha_{1} & \alpha_{2} \\ \beta_{1} & \beta_{2}\end{array}\right) \in \Re\left(\Gamma_{0}\right)\right.$, $k>1)$.

Lemma 2. Consider the set

$$
\mathfrak{U}=\left\{z \in \mathfrak{S}^{+}|z=x+i y,| x \mid \leqq C, y \geqq \xi\right\},
$$

where $C, \xi$ are positive constants. If $z=x+i y$ lies in $\mathfrak{u}$, then

$$
|c z+d| \geqq A|c i+d|
$$

for all real $c, d$, where $A$ depends only on $C$ and $\xi$. 
Proof. Disregarding the trivial case $c=0$, we first assume $\left|\frac{d}{c}\right|>2 C$. Then we have

$$
\begin{aligned}
\left|x+\frac{d}{c}\right| \geqq\left|\frac{d}{c}\right|-|x| \geqq\left|\frac{d}{c}\right|-C \\
>\left|\frac{d}{c}\right|-\frac{1}{2}\left|\frac{d}{c}\right|=\left|\frac{d}{2 c}\right| ;
\end{aligned}
$$

hence

$$
\begin{aligned}
|c z+d|^{2} & =c^{2}\left\{\left(x+\frac{d}{c}\right)^{2}+y^{2}\right\} \geqq \frac{d^{2}}{4}+\xi^{2} c^{2} \\
& \geqq A_{1}^{2}\left(c^{2}+d^{2}\right)=A_{1}^{2}|c i+d|^{2},
\end{aligned}
$$

where $A_{1}=\min \left\{\frac{1}{2}, \xi\right\}$. Therefore we have

$$
|c z+d| \geqq A_{1}|c i+d| \text {. }
$$

On the other hand, if $\left|\frac{d}{c}\right| \leqq 2 C$, we get

$$
\begin{aligned}
|c i+d|^{2} & =c^{2}+d^{2}=c^{2}\left(1+\left|\frac{d}{c}\right|^{2}\right) \\
& \leqq c^{2}\left(1+4 C^{2}\right)=c^{2} \frac{1+4 C^{2}}{\xi^{2}} \xi^{2} \\
& \leqq c^{2} \frac{1+4 C^{2}}{\xi^{2}}\left|z+\frac{d}{c}\right|^{2}=\frac{1+4 C^{2}}{\xi^{2}}|c z+d|^{2}
\end{aligned}
$$

hence

(5)

$$
|c z+d| \geqq \frac{\xi}{\sqrt{1+4 C^{2}}}|c i+d|
$$

By (4) and (5), we have

$$
|c z+d| \geqq A|c i+d|,
$$

where $A=\min \left\{A_{1}, \frac{\xi}{\sqrt{1+4 C^{2}}}\right\}$. This establishes the lemma.

Now we put

$$
\mathfrak{U}_{0}=\left\{z \in \mathfrak{S}^{+}|z=x+i y,| x \mid \leqq C, \xi \leqq y \leqq \xi^{\prime}\right\},
$$

where $C, \xi$ and $\xi^{\prime}$ denote positive constants; and we take coset representatives $\mathfrak{R}\left(\Gamma_{0}\right)$ such that each element in $\mathfrak{R}\left(\Gamma_{0}\right)$ satisfies the condition $|\log | L(i)||$ 
$\leqq \log \varepsilon_{0}^{2}$. Then, by Lemma 2, we have

$$
\begin{aligned}
\left|\alpha_{1} z+\alpha_{2}\right|^{-k}\left|\beta_{1} z+\beta_{2}\right|^{-k} & \leqq A^{-2 k}\left|\alpha_{1} i+\alpha_{2}\right|^{-k}\left|\beta_{1} i+\beta_{2}\right|^{-k} \\
& \leqq \frac{1}{2} A^{-2 k}\left\{\left|\alpha_{1} i+\alpha_{2}\right|^{-2 k}+\left|\beta_{1} i+\beta_{2}\right|^{-2 k}\right\}
\end{aligned}
$$

for all $z \in \mathfrak{U}_{0}$, and all $L=\left(\begin{array}{cc}\alpha_{1} & \alpha_{2} \\ \beta_{1} & \beta_{2}\end{array}\right) \in \mathfrak{\Re}\left(\Gamma_{0}\right)$. Hence we also have

$$
\sum_{L \in \Re\left(\Gamma_{0}\right)}\left|\alpha_{1} z+\alpha_{2}\right|^{-k}\left|\beta_{1} z+\beta_{2}\right|^{-k} \leqq \frac{1}{2} A^{-2 k}\left\{\sum_{L \in \Re\left(\Gamma_{0}\right)}\left|\alpha_{1} i+\alpha_{2}\right|^{-2 k}+\sum_{L \in \Re\left(\Gamma_{0}\right)}\left|\beta_{1} i+\beta_{2}\right|^{-2 k}\right\} .
$$

Convergence of the series $\sum_{L}\left|\beta_{1} i+\beta_{2}\right|^{-2 k}$ is reduced to the argument of $1^{\circ}$ putting $z_{0}=i$. And convergence of the series $\sum_{L}\left|\alpha_{1} i+\alpha_{2}\right|^{-2 k}$ is demonstrated in the following manner. We take $S=\left(\begin{array}{rr}0 & -1 \\ 1 & 0\end{array}\right) \in S L(2, \boldsymbol{R})$ and put $\Gamma_{0}^{\prime}=S \Gamma_{0} S^{-1}$. Then $\Gamma_{0}^{\prime}$ has the same genus and signature as $\Gamma_{0}$ and contains the hyperbolic element $S\left(\begin{array}{ll}\varepsilon_{0}^{2} & 0 \\ 0 & \varepsilon_{0}^{-2}\end{array}\right) S^{-1}$ which has fixed points $\{0, \infty\}$. Moreover the infinite cyclic group generated by $S\left(\begin{array}{ll}\varepsilon_{0}^{2} & 0 \\ 0 & \varepsilon_{0}^{-2}\end{array}\right) S^{-1}$ is given by $S Z_{0, \infty} S^{-1}$ and the set of right coset representatives $\Gamma_{0}^{\prime} \bmod S Z_{0, \infty} S^{-1}$ is given by $S \Re\left(\Gamma_{0}\right) S^{-1}$ to which a one-to-one correspondence with $\Re\left(\Gamma_{0}\right)$ is given. Since $S L S^{-1}=\left(\begin{array}{cc}\beta_{2} & -\beta_{1} \\ -\alpha_{2} & \alpha_{1}\end{array}\right)$ and $\left|\alpha_{1} i+\alpha_{2}\right|^{-2 k}=\left|-\alpha_{2} i+\alpha_{1}\right|^{-2 k}$ for all $L=\left(\begin{array}{cc}\alpha_{1} & \alpha_{2} \\ \beta_{1} & \beta_{2}\end{array}\right)$, we know that

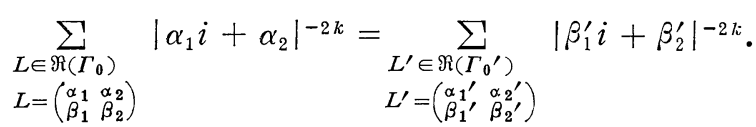

Because of $|\log | L^{\prime}(i)||=|\log | L(i)|| \leqq \log \varepsilon_{0}{ }^{2}$, convergence of the right hand side is reduced to the argument of $1^{\circ}$ putting $z_{0}=i$.

Finally, let $K$ be any compact set in $\mathfrak{S}^{+}$and take $\mathfrak{H}_{0}$ a rectangle such that $\mathfrak{U}_{0} \supseteq K$. Then the series

$$
\begin{aligned}
& \sum_{L \in \Re\left(\Gamma_{0}\right)}\left(\alpha_{1} z+\alpha_{2}\right)^{-k}\left(\beta_{1} z+\beta_{2}\right)^{-k} \\
& L=\left(\begin{array}{ll}
\alpha_{1} & \alpha_{2} \\
\beta_{1} & \beta_{2}
\end{array}\right)
\end{aligned}
$$

converges absolutely, uniformly over $K$, since it does so over $\mathfrak{U}_{0}$ as mentioned above.

$3^{\circ}$ Since $0<\arg L(z)<\pi$ for all $L \in \Re\left(\Gamma_{0}\right)$, we have

$$
\left|e^{2 \pi i \nu \frac{\log L(z)}{\log \varepsilon_{0}}}\right| \leqq \begin{cases}1 & , \text { if } \nu \geqq 0, \\ e^{-\frac{\nu \pi^{2}}{2 \log \varepsilon_{0}}} & , \text { if } \nu<0 .\end{cases}
$$


for all $L \in \Re\left(\Gamma_{0}\right)$. Obviously each $e^{2 \pi i \nu \frac{\log L(z)}{\log \varepsilon_{0}}}\left(L \in \mathfrak{R}\left(\Gamma_{0}\right)\right)$ is holomorphic in $\mathfrak{S}^{+}$.

Sumarizing these results ((i) and (ii)) we obtain the result that if $k>1,4)$ then the series $\Xi_{-2 k}\left(z ; \Gamma_{0}, \nu\right)$ is a holomorphic automorphic form of dimension $-2 k$ for the group $\Gamma_{0}$ and is the so-called hyperbolic Poincare series with respect to $\sigma=\left(\begin{array}{ll}\varepsilon_{0}^{2} & 0 \\ 0 & \varepsilon_{0}^{-2}\end{array}\right)$.

\section{\$4. Inner product formula.}

Let $f(z)$ be any form in $\Im_{2 k}\left(\Gamma_{0}\right)$. Then $f(z)$ has the following HFE. with respect to $\sigma$ :

$$
f(z)=z^{-k} \sum_{n=-\infty}^{\infty} c_{n} z^{\pi i \frac{n}{2 \log \varepsilon_{0}}}
$$

In the following, we calculate the Petersson inner product of $f(z)$ with $\Xi_{-2 k}\left(z ; \Gamma_{0}, \nu\right)$, i.e.,

(6) $\left(f(z), \Xi_{-2 k}(z ; \nu)\right)=(f, \Xi)=\iint_{D} f(z) \overline{\Xi_{-2 k}(z ; \nu)} y^{2 k-2} d x d y, \quad z=x+i y$

where $D$ denotes a compact fundamental domain of $\Gamma_{0}$ and $\overline{\Xi_{-2 k}(z ; \nu)}$ stands for the complex conjugate of $\Xi_{-2 k}(z ; \nu)$. If $k>1$, the integral (6) does converge and obviously the inner product (6) is independent of the choice of $D$. From now on we assume that $k>1$.

Now we put

$$
\vartheta_{l}(z)=z^{-k+\pi i \frac{l}{2 \log \varepsilon_{0}}}
$$

Then we have

$$
\begin{aligned}
\Xi_{-2 k}(z ; \nu) & =\sum_{L \in \Re\left(\Gamma_{0}\right)}(L(z))^{-k+\pi i \frac{\nu}{2 \log \varepsilon_{0}}\left(\beta_{1} z+\beta_{2}\right)^{-2 k}} \\
& =\sum_{L \in \Re\left(\Gamma_{0}\right)} \vartheta_{\nu}(z) \mid L,
\end{aligned}
$$

and

$$
f(z)=\sum_{n=-\infty}^{\infty} c_{n} \vartheta_{n}(z)
$$

Because of $Z_{0, \infty} \nRightarrow\left(\begin{array}{rr}-1 & 0 \\ 0 & -1\end{array}\right)$, the system $\Re\left(\Gamma_{0}\right)$ contains both $L$ and $-L$, and these matrices make the same contribution to the integral. Thus, putting

4) In the case of $k=1$, cf. Petersson [7]. 
$\Re^{*}\left(\Gamma_{0}\right)=\Re\left(\Gamma_{0}\right) /\left\{ \pm 1_{2}\right\}^{5)}$, we have

$$
(f, \Xi)=2 \iint_{D} f(z) \sum_{L \in \Re^{*}\left(\Gamma_{0}\right)} \overline{\vartheta_{\nu}(z) \mid L} y^{2 k-2} d x d y
$$

Since the series $Z_{-2 k}(z ; \nu)$ converges absolutely, uniformly over $D$,

$$
\begin{aligned}
(f, \Xi) & =2 \sum_{L \in \Re^{*}\left(\Gamma_{0}\right)} \iint_{D} f(z) \overline{\vartheta_{\nu}(z) \mid L} y^{2 k-2} d x d y \\
& =2 \sum_{L \in \Re^{*}\left(\Gamma_{0}\right)} \iint_{L D} f(z) \overline{\vartheta_{\nu}(z)} y^{2 k-2} d x d y \\
& =2 \iint_{\Re^{*}\left(\Gamma_{0}\right) D} f(z) \overline{\vartheta_{\nu}(z)} y^{2 k-2} d x d y .
\end{aligned}
$$

Because of $\mathfrak{L}^{+}=\Gamma_{0} D=Z_{0, \infty} \Re\left(\Gamma_{0}\right) D$, it follows that $: \AA^{*}\left(\Gamma_{0}\right) D$ is a fundamental domain for $Z_{0, \infty}$, the group generated by $\sigma$. We have remarked in $\S 3$ that the series $\Xi_{-2 k}(z ; \nu)$ is independent of the system $\Re\left(\Gamma_{0}\right)$ of right coset representatives $\Gamma_{0} \bmod Z_{0, \infty}$. Thus we can assume that $\Re\left(\Gamma_{0}\right)$ was chosen in such a way that

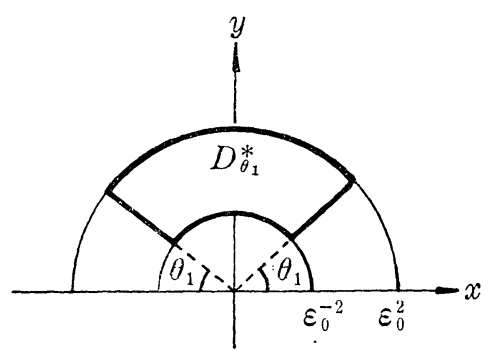

Fig. 1

$$
\left\{\begin{array}{l}
D_{\theta_{1}}^{*}: \varepsilon_{0}^{-4} \leqq x^{2}+y^{2} \leqq \varepsilon_{0}^{4}, \theta_{1} \leqq \arg z \leqq \pi-\theta_{1}\left(0<\theta_{1}<\frac{\pi}{2}\right), \\
\Re\left(\Gamma_{0}\right) D=\lim _{\theta_{1} \rightarrow+0} D_{\theta_{1}}^{*} . \quad \text { (consult Figure 1) }
\end{array}\right.
$$

Then we have

$$
(f, \Xi)=2 \lim _{\theta_{1} \rightarrow+0} \iint_{D_{\theta_{1}}^{*}} \sum_{\substack{* \\ *}}^{\infty} c_{n} \vartheta_{n}(z) \overline{\vartheta_{\nu}(z)} y^{2 k-2} d x d y
$$

and since $\sum_{n}$ converges uniformly over the closed domain $D_{\theta_{1}}^{*}$,

5) $1_{2}$ means the identity matrix of degree 2 . 


$$
(f, \Xi)=2 \lim _{\theta_{1} \rightarrow+0} \sum_{n=-\infty}^{\infty} c_{n} \iint_{D_{\theta_{1}}^{*}} \vartheta_{n}(z) \overline{\vartheta_{\nu}(z)} y^{2 k-2} d x d y .
$$

Now we put $\log z=u+i v$. Then

$$
\begin{gathered}
y^{2 k-2}|J(u, v)|=e^{2 k u} \sin ^{2 k-2} v, \\
\vartheta_{n}(z) \overline{\vartheta_{\nu}(z)}=e^{-2 k u-\pi(n+\nu) \frac{v}{2 \log \varepsilon_{0}}+i \pi(n-\nu) \frac{u}{2 \log \varepsilon_{0}},}
\end{gathered}
$$

where $J(u, v)$ denotes the Jacobian of $(x, y)$ with respect to $(u, v)$. Therefore we obtain

$$
(f, \Xi)=2 \lim _{\theta_{1} \rightarrow+\infty} \sum_{n=-\infty}^{\infty} c_{n} \int_{\theta_{1}}^{\pi-\theta_{1}} \int_{-2 \log \varepsilon_{0}}^{2 \log \varepsilon_{0}} e^{-\pi(n+\nu) \frac{v}{2 \log \varepsilon_{0}}+i \pi(n-\nu) \frac{u}{2 \log \varepsilon_{0}}} \sin ^{2 k-2} v d u d v .
$$

The inside integral vanishes unless $n=\nu$; hence

$$
\begin{aligned}
(f, \Xi) & =2 \lim _{\theta_{1} \rightarrow+0} c_{\nu} \int_{-2 \log \varepsilon_{0}}^{2 \log \varepsilon_{0}} d u \int_{\theta_{1}}^{\pi-\theta_{1}} e^{-\nu \pi \frac{v}{\log \varepsilon_{0}}} \sin ^{2 k-2} v d v \\
& =8 \log \varepsilon_{0} \int_{0}^{\pi} e^{-\nu \pi \frac{v}{\log \varepsilon_{0}}} \sin ^{2 k-2} v d v \cdot c_{\nu} \\
& =\varepsilon_{2 k}(\nu) \cdot c_{\nu} .
\end{aligned}
$$

Appling Kummer formulas [4, p. 217, (31) and (32)], $\varepsilon_{2 k}(\nu)$ can be calculated as follows:

$$
\varepsilon_{2 k}(\nu)=2^{5-2 k} \pi \log \varepsilon_{0} e^{-\frac{\pi^{2} \nu}{2 \log \varepsilon_{0}}} \frac{\Gamma(2 k-1)}{\Gamma\left(k+\frac{\pi \nu}{2 \log \varepsilon_{0}} i\right) \Gamma\left(k-\frac{\pi \nu}{2 \log \varepsilon_{0}} i\right)} .
$$

Therefore we have the following inner product formula

$$
\begin{aligned}
\left(f(z), z_{-2 k}\left(z ; \Gamma_{0}, \nu\right)\right)= & 2^{5-2 k} \pi \log \varepsilon_{0} e^{-\frac{\pi^{2} \nu}{2 \log \varepsilon_{0}}} \Gamma(2 k-1) \cdot c_{\nu} \times \\
& \times \frac{1}{\Gamma\left(k+\frac{\pi \nu}{2 \log \varepsilon_{0}} i\right) \Gamma\left(k-\frac{\pi \nu}{2 \log \varepsilon_{0}} i\right)} .
\end{aligned}
$$

This formula has been obtained by applying the general theory of Petersson [6] to the case of our group $\Gamma_{0}$.

\section{§. Hyperbolic Fourier coefficients.}

Given a group $\Gamma^{p \times q}$ as described in $\S 1$, and let $f(z)$ be any element in the image $T\left(S_{k}(\Gamma(\sqrt{p}))\right)$ of the space $S_{k}(\Gamma(\sqrt{p}))$ by the linear map $T$. 
Then, for each form $f(z)$, there exists some element $F(\boldsymbol{z})$ in $S_{k}(\Gamma(\sqrt{p}))$ such that $T(F(\boldsymbol{z}))=f(z)$ and each of them has the Fourier expansion

$$
\begin{aligned}
F(\boldsymbol{z})= & \sum_{\substack{\mu: \text { integers in } F \\
\mu \equiv 0 \bmod \left(\frac{1}{\sqrt{p}}\right)}} c_{\mu} e^{2 \pi i \operatorname{tr}(\mu \boldsymbol{z})} \\
\mu>0 \text { or } \mu=0 &
\end{aligned}
$$

and

$$
f(z)=z^{-k} \sum_{n=-\infty}^{\infty} c_{n} z^{\pi i \frac{n}{2 \log \varepsilon_{0}}},
$$

respectively. The purpose of this section is to express the hyperbolic Fourier coefficients $c_{n}$ by the formula contained the parabolic Fourier coefficients $c_{\mu}$ of $F(\boldsymbol{z})$.

Because of $f(z)=T(F(z)), f(z)$ has also the following expansion:

$$
f(z)=z^{-k} g(z), \quad g(z)=\sum_{\mu} c_{\mu} e^{2 \pi i\left(\mu z-\bar{\mu} \frac{1}{q z}\right)}
$$

and then

$$
\begin{aligned}
\left(f(z), \Xi_{-2 k}(z ; \nu)\right) & =\left(z^{-k} \sum_{n=-\infty}^{\infty} c_{n} z^{\pi i \frac{n}{2 \log \varepsilon_{0}}}, \Xi_{-2 k}(z ; \nu)\right) \\
& =\left(z^{-k} \sum_{\mu} c_{\mu} e^{2 \pi i\left(\mu z-\bar{\mu} \frac{1}{q z}\right)}, \Xi_{-2 k}(z ; \nu)\right) .
\end{aligned}
$$

The former has been calculated in $\S 4$ and in the following, we shall calculate the latter. By the same method as in $\S 4$, we first have

$$
\left.\left(z^{-k} g(z), \Xi_{-2 k}(z ; \nu)\right)=2 \lim _{\theta_{1} \rightarrow+0} \sum_{\mu} c_{\mu} \iint_{D_{\theta_{1}}^{*}} e^{2 \pi i(\mu z-\bar{\mu}} \frac{1}{q z}\right) z^{-k} \bar{z}^{-k-\pi i} \frac{\nu}{2 \log \varepsilon_{0}} y^{2 k-2} d x d y,
$$

where $D_{\theta_{1}}^{*}$ is as shown in Figure 2:

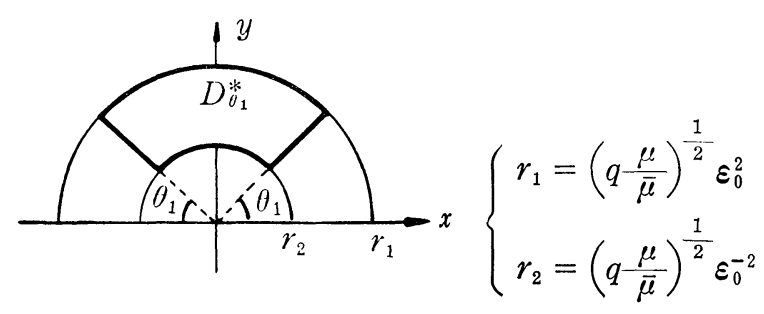

Fig. 2 
Here if we put

$$
z=\left(\frac{\bar{\mu}}{q \mu}\right)^{\frac{1}{2}} w, w=u+i v,
$$

then

$$
\begin{aligned}
\left(z^{-k} g(z), \Xi\right) & =2 q^{\pi i \frac{\nu}{4 \log \varepsilon_{0}}} \lim _{\theta_{1} \rightarrow+0} \sum_{\mu}\left(\frac{\mu}{\bar{\mu}}\right)^{\frac{\pi i}{\log \varepsilon_{0}} \frac{\nu}{4}} c_{\mu} \times \\
& \times \iint_{D_{\theta_{1}}^{* * *}} e^{2 \pi i\left(\frac{N(\mu)}{q}\right)^{\frac{1}{2}}\left(w-\frac{1}{w}\right)} w^{-k} \bar{w}^{-k-\pi i \frac{\nu}{2 \log \varepsilon_{0}}} v^{2 k-2} d u d v .
\end{aligned}
$$

As for $D_{\theta_{1}}^{* *}$, consult Figure 3:

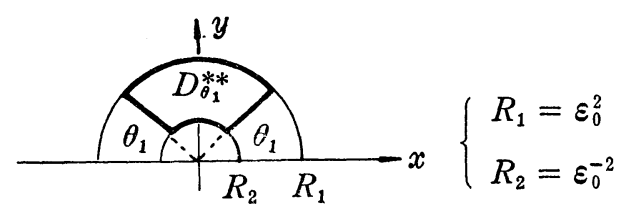

Fig. 3

It is well known that

$$
e^{\frac{t}{2}\left(z-\frac{1}{z}\right)}=\sum_{n=-\infty}^{\infty} J_{n}(t) z^{n},|z|<\infty
$$

where $J_{n}(t)$ denotes the Bessel function of order $n$ and argument $t$ of the first kind.6) Therefore we have

$$
\begin{aligned}
\left(z^{-k} g(z), \Xi\right)= & 2 q^{\pi i \frac{\nu}{4 \log \varepsilon_{0}}} \lim _{\theta_{1} \rightarrow+0} \sum_{\mu}\left(\frac{\mu}{\bar{\mu}}\right)^{\frac{\pi i}{\log \varepsilon_{0}} \frac{\nu}{4}} c_{\mu} \times \\
& \times \sum_{n=-\infty}^{\infty} J_{n}\left(4 \pi i\left(\frac{N(\mu)}{q}\right)^{\frac{1}{2}}\right) \iint w^{n-k} \bar{w}^{-k-\pi i \frac{\nu}{2 \log \varepsilon_{0}}} v^{2 k-2} d u d v .
\end{aligned}
$$

Here if we put again $w=R e^{i \theta}$, then

$$
\begin{aligned}
& \iint_{D_{1}^{* *}} w^{n-k} \bar{w}^{-k-\pi i \frac{\nu}{2 \log \varepsilon_{0}}} v^{2 k-2} d u d v \\
& =\int_{\varepsilon_{0}^{-2}}^{\varepsilon_{0}^{2}} R^{n-\pi i \frac{\nu}{2 \log \varepsilon_{0}}-1} d R \int_{\theta_{1}}^{\pi-\theta_{1}} e^{i\left(n+\pi i \frac{\nu}{2 \log \varepsilon_{0}}\right) \theta} \sin ^{2 k-2} \theta d \theta
\end{aligned}
$$

6) For instance, cf. Watson [9. p. 14]. 


$$
=\frac{(-1)^{\nu}}{n-\pi i \frac{\nu}{2 \log \varepsilon_{0}}}\left(\varepsilon_{0}^{2 n}-\varepsilon_{0}^{-2 n)} \int_{\theta_{1}}^{\pi-\theta_{1}} e^{i\left(n+\pi i \frac{\nu}{2 \log \varepsilon_{0}}\right) \theta} \sin ^{2 k-2} \theta d \theta\right.
$$

and since $\sum_{\mu}$ and $\sum_{n}$ are uniformly for $\theta_{1}$, we have

$$
\begin{aligned}
& \left(z^{-k} g(z), \quad \Xi\right)=2(-1)^{\nu} q^{\pi i \frac{\nu}{4 \log \varepsilon_{0}}} \sum_{\mu}\left(\frac{\mu}{\bar{\mu}}\right)^{\frac{\pi i}{\log \varepsilon_{0}} \frac{\nu}{4}} c_{\mu} \times \\
& \quad \times \sum_{n=-\infty}^{\infty} \frac{\varepsilon_{0}^{2 n}-\varepsilon_{0}^{-2 n}}{n-\pi i \frac{\nu}{2 \log \varepsilon_{0}}} J_{n}\left(4 \pi i\left(\frac{N(\mu)}{q}\right)^{\frac{1}{2}}\right) \int_{0}^{\pi} e^{\left(-\pi \frac{\nu}{2 \log \varepsilon_{0}}+i n\right) \theta} \sin ^{2 k-2} \theta d \theta .
\end{aligned}
$$

Applying Kummer formulas, the integral can be calculated as follows:

$$
\begin{gathered}
\int_{0}^{\pi} e^{\left(-\pi \frac{\nu}{2 \log \varepsilon_{0}}+i n\right) \theta} \sin ^{2 k-2} \theta d \theta \\
=2^{2(1-k)} \pi e^{\frac{\pi}{2}\left(-\pi \frac{\nu}{2 \log \varepsilon_{0}}+i n\right)} \frac{\Gamma(2 k-1)}{\Gamma\left(k+\frac{n}{2}+\pi i \frac{\nu}{4 \log \varepsilon_{0}}\right) \Gamma\left(k-\frac{n}{2}-\pi i \frac{\nu}{4 \log \varepsilon_{0}}\right)} .
\end{gathered}
$$

Summing up, we obtain

$$
\begin{aligned}
& \left(z^{-k} \sum_{\mu} c_{\mu} e^{2 \pi i\left(\mu z-\bar{\mu} \frac{1}{q z}\right)}, \Xi_{-2 k}(z ; \nu)\right) \\
& =(-1)^{\nu} 2^{3-2 k} \pi q^{\pi i \frac{\nu}{4 \log \varepsilon_{0}}} e^{-\frac{\pi^{2} \nu}{4 \log \varepsilon_{0}}} \Gamma(2 k-1) \sum_{\mu}\left(\frac{\mu}{\bar{\mu}}\right)^{\frac{\pi i}{\log \varepsilon_{0}} \frac{\nu}{4}} c_{\mu} \times \\
& \times \sum_{n=-\infty}^{\infty} i^{n} \frac{\varepsilon_{0}^{2 n}-\varepsilon_{0}^{-2 n}}{n-\pi i \frac{\nu}{2 \log \varepsilon_{0}}} J_{n}\left(4 \pi i\left(\frac{N(\mu)}{q}\right)^{\frac{1}{2}}\right) \times \\
& \times \frac{1}{\Gamma\left(k+\frac{n}{2}+\pi i \frac{\nu}{4 \log \varepsilon_{0}}\right) \Gamma\left(k-\frac{n}{2}-\pi i \frac{\nu}{4 \log \varepsilon_{0}}\right)} .
\end{aligned}
$$

It is now clear that the above formula (8), combined with the formula (7) in $\S 4$, proves the following theorem which is announced in $\S 0$ :

TheOrem. The notations and the assumptions being as above, we obtain

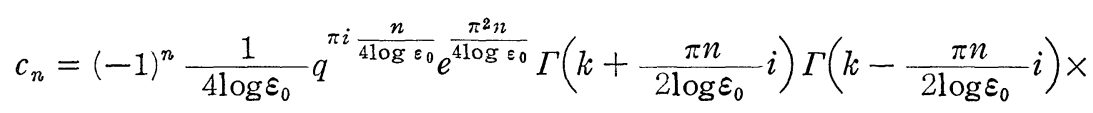




$$
\begin{aligned}
& \times \sum_{\substack{\mu: \text { integers in } Q(\sqrt{p}) \\
\mu \equiv 0 \bmod \left(\frac{1}{\sqrt{p}}\right) \\
\mu>0 \text { or } \mu=0}}\left(\frac{\mu}{\bar{\mu}}\right)^{\frac{\pi i}{\log \varepsilon_{0}} \frac{n}{4}} c_{\mu} \sum_{\nu=-\infty}^{\infty} i^{\nu} \frac{\varepsilon_{0}{ }^{2 \nu}-\varepsilon_{0}^{-2 \nu}}{\nu-\pi i \frac{n}{2 \log \varepsilon_{0}}} J_{\nu}\left(4 \pi i\left(\frac{N(\mu)}{q}\right)^{\frac{1}{2}}\right) \times \\
& \times \frac{1}{\Gamma\left(k+\frac{\nu}{2}+\pi i \frac{n}{4 \log \varepsilon_{0}}\right) \Gamma\left(k-\frac{\nu}{2}-\pi i \frac{n}{4 \log \varepsilon_{0}}\right)}
\end{aligned}
$$

\section{REFERENCES}

[1] M. Eichler, La theorie des corréspondances des corps des fonctions algébriques et leurs applications dans L'arithmetiques, Lecture Notes, Nancy, 1954.

[2] - Lectures on modular correspondences, Tata Institute, Bombay, 1955-56.

[ 3 ] K.B. Gundlach, Die Fixpunkte einiger Hilbertscher Modulgruppen, Math. Ann. 157 369-390.

[4] E.E. Kummer, De integralibus definitis et seriebus infinitis, J. reine angew. Math. 17 (1837), 210-227.

[5] H. Petersson, Zur analytischen Theorie der Grenzkreisgruppen V, Math. Z. 44(1939), $127-155$.

[6] - Einheitliche Begrundung der Vollstandigkeitssatze fur der Poincaréschen Reihen von reeller Dimension bei beliebigen Grenzkreisgruppen von erste Art, Abh. der Hamburg Univ. 14(1941), 22-60.

[ 7 ] — Ein Summationsverfahren fur die Poincaréschen Reihen von der Dimension -2 zu den hyperbolischen Fixpunktepaaren, Math. Z. 49(1944), 441-496.

[ 8 ] H. Shimizu, On discontinuous groups operating on the product of the upper half plane, Ann. of Math. 77(1963), 33-71.

[9] G.N. Watson, A treatise on the theory of Bessel functions, 2nd edition, Cambridge, 1962.

College of General Education, Osaka University 